\section{Late dumping syndrome or postprandial reactive hypoglycaemic syndrome after bariatric surgery}

\author{
Frederique Van de Velde and Bruno Lapauw
}

In the August 2020 issue of Nature Reviews Endocrinology, a group of researchers proposed an international consensus on the diagnosis and management of dumping syndrome (Scarpellini, E. et al. Nat. Rev. Endocrinol. 16, $\left.448-466(2020)^{1}\right)$. On the basis of the limited evidence available, the authors made efforts to fill a gap in clinical guidance on how to deal with these complications that sometimes severely impact the life of patients. However, we feel that some of the recommendations are problematic.

Firstly, they propose the use of 'late dumping syndrome' to describe the occurrence of hypoglycaemic symptoms in these patients. This terminology, however, is misleading as these symptoms and underlying pathophysiology, albeit incompletely understood, are not related to mechanical or physical processes whereby ingested nutrients are 'dumped'. Instead, symptoms are probably related to changes in postprandial absorption of carbohydrates and associated hormonal responses in incretin and insulin release. Therefore, we suggest using the term 'postprandial reactive (hypoglycaemic) syndrome' instead.

Secondly, the authors recommend that, to diagnose 'late dumping syndrome', patients should have glucose levels $<2.8 \mathrm{mmol} / \mathrm{l}$ during a modified oral glucose tolerance test (OGTT). However, it is not unequivocally established that symptoms in these patients are strictly related to low blood levels of glucose as such. Indeed, some studies have found that hypoglycaemic values during an OGTT correlate with hypoglycaemic symptoms such as drowsiness in patients who have undergone bariatric surgery ${ }^{2}$; however, other studies could not confirm this finding ${ }^{3}$. One study even showed that people with 'late dumping syndrome' are more accurately recognized by their subjective (for example, perspiration or hunger) than objective (for example, nadir glucose values) symptoms during an OGTT ${ }^{4}$. Moreover, a low level of glucose during an OGTT is not a specific finding. For instance, a study showed that about $10 \%$ of patients without any hypoglycaemic symptoms had nadir glucose levels of $\leq 2.7 \mathrm{mmol} / \mathrm{l}$ during an OGTT $^{5}$. Another study even reported that $72 \%$ of their post-bariatric surgery cohort had serum levels of glucose $\leq 3.3 \mathrm{mmol} / 1$ during OGTT $^{3}$. As such, using such a nonphysiologically provocative test (which also has no reliable evidence for high reproducibility and sensitivity, as mentioned by Scarpellini et al. ${ }^{1}$ ) will lead to many false positive results, overdiagnosis and the risk of overtreatment. Instead, research efforts should be made to evaluate alternative tests, such as a standardized (solid) mixed meal test or continuous glucose monitoring if glycaemic patterns suggestive of hypoglycaemic syndrome are identified in these patients.

Finally, and perhaps most relevant in clinical practice, is that one test to diagnose two different entities might seem efficient but probably is not. Indeed, a substantial proportion of patients examined for 'late dumping
We read with great interest the comments of Van de Velde and Lapauw on our evidencebased guideline (International consensus on the diagnosis and management of dumping syndrome. Nat. Rev. Endocrinol. 16, 448-466 $\left.(2020)^{1}\right)$. The authors raise a number of issues related to the recommendations of an international consensus panel (Late dumping syndrome or postprandial reactive hypoglycaemic syndrome after bariatric surgery. Nat. Rev. Endocrinol. https://doi.org/10.1038/ s41574-021-00473-6 (2021) 2).

They suggest the terminology 'late dumping syndrome' should be abandoned and replaced by 'postprandial reactive hypoglycaemic syndrome. In our opinion, narrowing down the chain of events to reactive hypoglycaemia is an undesirable simplification. Indeed, early postprandial hyperglycaemia is a prerequisite for late hypoglycaemia ${ }^{1,3-5}$. The observation that early hyperglycaemia is accompanied syndrome' will experience symptoms of early dumping upon OGTT, and vice versa, further driving medical overdiagnosis in this already troubled patient population.

There is a reply to this letter by Scarpellini, E., Arts, J., Vanuytsel, T. \& Tack, J. Nat. Rev. Endocrinol. https://doi.org/10.1038/s41574021-00474-5 (2021).

\section{Frederique Van de Velde ${ }^{\varpi}$ and Bruno Lapauw Department of Endocrinology, Ghent University Hospital, Ghent, Belgium. \\ 凶e-mail: frederique.vandevelde@ugent.be https://doi.org/10.1038/s41574-021-00473-6}

\footnotetext{
Scarpellini, E. et al. International consensus on the diagnosis and management of dumping syndrome. Nat. Rev. Endocrinol. 16, 448-466 (2020).

2. Arts, J. et al. Efficacy of the long-acting repeatable formulation of the somatostatin analogue octreotide in postoperative dumping. Clin. Gastroenterol. Hepatol. 7, 432-437 (2009).

3. Roslin, M. et al. Abnormal glucose tolerance testing following gastric bypass demonstrates reactive hypoglycemia. Surg. Endosc. 25, 1926-1932 (2011).

4. Van der Kleij, F. G. H., Vecht, J., Lamers, C. B. H. W. $\&$ Masclee, A. A. M. Diagnostic value of dumping provocation in pations after gastric surgery. Scand J. Gastroenterol. 31, 1162-1166 (1996).

5. Lev-Ran, A. \& Anderson, R. W. The diagnosis of postprandial hypoglycemia. Diabetes 30, 996-999 (1981).
}

Competing interests

The authors declare no competing interests.

\title{
Reply to: Late dumping syndrome or postprandial reactive hypoglycaemic syndrome after bariatric surgery
}

\author{
Emidio Scarpellini, Joris Arts, Tim Vanuytsel and Jan Tack (iD)
}

by a rise in pulse rate and haematocrit ${ }^{1,3,4}$, as well as the therapeutic benefit of acarbose, the SGLT2 inhibitor empagliflozin and agents that increase meal viscosity (such as guar gum, pectin and glucomannan $)^{6}$, supports the sequence of events where initial overload of the small bowel with nutrients, including carbohydrates, triggers late hypoglycaemia through excessive release of insulin and incretins. Several cohort studies of patients who have undergone bariatric surgery confirm the association between (early) dumping syndrome and hypoglycaemia ${ }^{5,7-9}$. The idiom dumping syndrome, with its early and late component, covers this sequence.

The authors query the proposed threshold of glycaemia $<2.8 \mathrm{mmol} / 1$ during a modified oral glucose tolerance test (OGTT), as this might occur after a challenge in healthy controls ${ }^{6}$. The evidence-based guideline only advocates performing OGTT in patients with 